\title{
Process Tomography of Field Damping and Measurement of Fock State Lifetimes by Quantum Nondemolition Photon Counting in a Cavity
}

\author{
M. Brune, ${ }^{1, *}$ J. Bernu, ${ }^{1}$ C. Guerlin, ${ }^{1, \dagger}$ S. Deléglise,${ }^{1}$ C. Sayrin, ${ }^{1}$ S. Gleyzes, ${ }^{1 ;}$ S. Kuhr,${ }^{1, \S}$ I. Dotsenko,,${ }^{1,2}$ \\ J. M. Raimond, ${ }^{1}$ and S. Haroche ${ }^{1,2}$ \\ ${ }^{1}$ Laboratoire Kastler Brossel, Ecole Normale Supérieure, CNRS, Université P. et M. Curie, \\ 24 rue Lhomond, F-75231 Paris Cedex 05, France \\ ${ }^{2}$ Collège de France, 11 Place Marcelin Berthelot, F-75231 Paris Cedex 05, France
}

(Received 8 September 2008; published 8 December 2008)

\begin{abstract}
The relaxation of a quantum field stored in a high- $Q$ superconducting cavity is monitored by nonresonant Rydberg atoms. The field, subjected to repetitive quantum nondemolition photon counting, undergoes jumps between photon number states. We select ensembles of field realizations evolving from a given Fock state and reconstruct the subsequent evolution of their photon number distributions. We realize in this way a tomography of the photon number relaxation process yielding all the jump rates between Fock states. The damping rates of the $n$ photon states $(0 \leq n \leq 7)$ are found to increase linearly with $n$. The results are in excellent agreement with theory including a small thermal contribution.
\end{abstract}

PACS numbers: 03.65.Yz, 03.65.Ta, 42.50.Pq

Quantum process tomography aims at determining experimentally the superoperator describing the evolution of a quantum system's density matrix [1]. This information is acquired by preparing a set of test states and monitoring their evolution. The method has been applied so far to spins in NMR experiments [2], to solid state qubits [3], to vibrational states of atoms [4] and to quantum gate operations $[5,6]$. We apply here process tomography to the photon number distribution of a relaxing field stored in a high- $Q$ superconducting cavity $C$, in which Fock states are used as test states.

Cavity field relaxation is described by a rate equation which, restricted to the photon number distribution $P(n, t)$, is:

$$
\frac{d P(n, t)}{d t}=\sum_{n^{\prime}} K_{n, n^{\prime}} P\left(n^{\prime}, t\right)
$$

A general model (linear coupling to a Markovian bath of oscillators) leads to [7] $K_{n, n}=-\kappa\left[\left(1+n_{b}\right) n+n_{b}(n+\right.$ 1)], $K_{n, n+1}=\kappa\left(1+n_{b}\right)(n+1), K_{n, n-1}=\kappa n_{b} n$, all the other coefficients being 0 . Here, $\kappa$ is the field energy damping rate and $n_{b}$ the mean number of blackbody photons at temperature $T$. The lifetime of the $n$-Fock state is $-1 / K_{n, n}$. We report here a complete experimental determination of the $K_{n, n^{\prime}}$ coefficients.

Our experiment relies on a quantum nondemolition (QND) procedure [8] counting the number $n$ of photons stored in $C$, based on the measurement of cavity-fieldinduced light shifts on Rydberg atoms crossing $C$ one by one. It projects the field onto Fock states with high fidelity. By detecting long sequences of QND probe atoms along single field realizations, we follow the field evolution and observe the jumps between Fock states due to damping $[9,10]$. By analyzing an ensemble of field trajectories, we partially reconstruct the superoperator describing field relaxation and measure the lifetimes of individual Fock states, scaling as $1 / n$ at zero temperature [11]. This Letter provides insights into the physics of these highly nonclassical states whose production by random $[9,10]$ or deterministic [12] processes has recently been demonstrated.

Our setup $[9,13]$ is depicted in Fig. 1(a). The high- $Q$ superconducting cavity $C$, operating at $51 \mathrm{GHz}$, has a damping time $T_{c}=1 / \kappa=0.130 \mathrm{~s}$ [14]. A pulsed microwave source $S$, coupled by diffraction on the mirrors' edges, can inject into $C$ a coherent field. The cavity field is probed by a pulsed monokinetic stream $(v=250 \mathrm{~m} / \mathrm{s})$ of rubidium atoms excited in box $B$ to the circular Rydberg state $g$ (principal quantum number 50). Before $C$, the
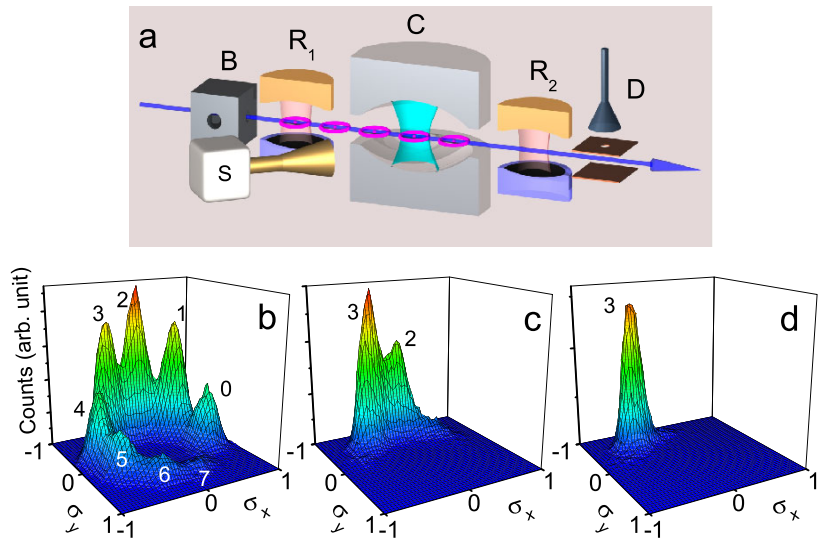

FIG. 1 (color online). (a) Scheme of experimental setup. (b) Histogram of the transverse atomic pseudospin after interaction with a coherent field in $C$ (initial mean photon number 4.4). Photon numbers associated to each peak are given. (c) Spin histogram after selection of the $n=3$ Fock state. The $n=2$ peak is due to photon loss between selection and measurement. (d) Same histogram as in (c) conditioned to a post-selection measurement excluding events in which a photon is lost. 
atoms experience in the low- $Q$ cavity $R_{1}$ a $\pi / 2$ pulse resonant on the transition to level $e$ (circular state with principal quantum number 51). The atoms enter $C$ in the superposition $(|e\rangle+|g\rangle) / \sqrt{2}$. They undergo nonresonant light shifts in $C$, resulting in a phase shift $\Phi(n)$ of the atomic superposition which is, to first order, linear in $n$. The phase shift per photon is set to $\Phi_{0} \simeq \pi / 4$.

We consider fields with a small probability of having $n>7$. In the Bloch pseudospin representation, the atomic state at the exit of $C$ points along one out of 8 directions equally distributed in the equatorial plane of the Bloch sphere, corresponding to values of $n$ varying from 0 (axis $O x$ ) to 7. After leaving $C$, the atoms are submitted to a second $\pi / 2$ pulse in $R_{2}$ with an adjustable phase $\phi$ with respect to that of $R_{1}$. The atoms are detected by the field ionization counter $D$ discriminating the states $e$ and $g$. This detection amounts to measuring the atomic spin at the exit of $C$ in the direction at an angle $\phi$ with $O x$ in the equatorial plane of the Bloch sphere. On average, we detect one atom every $\tau=0.24 \mathrm{~ms}\left(T_{c} / \tau=540\right.$ atoms detected during $T_{c}$ ).

Figure 1(b) shows a 3D histogram of the transverse atomic spin (components $\sigma_{x}$ and $\sigma_{y}$ ) after interaction with a coherent field in $C$. Each point in the Bloch sphere equatorial plane is obtained by measuring the average value of spin projections, on a sample of 110 consecutive atoms crossing $C$ in a $110 \tau=26 \mathrm{~ms}$ time interval, much shorter than $T_{c}$. About 700 atoms are sent across $C$, out of which we extract $\simeq 600$ atomic samples of 110 consecutive atoms. The procedure is repeated 2000 times, yielding about $10^{6}$ spin measurements. The histogram clearly shows that the direction of the atomic spin is quantized.

After a measurement indicating a spin pointing towards a peak of this histogram, the field is projected onto the corresponding Fock state. This is checked by correlating two independent successive samples of 110 atoms along a single field realization. The first pins down $n$ and the second remeasures it. Figure 1(c) shows the histogram of second measurements after selection of $n=3$. It exhibits a main $n=3$ peak with an $n=2$ satellite due to field relaxation during the $26 \mathrm{~ms}$ time delay between the two measurements. This satellite can be suppressed by postselection. Figure 1(d) displays the histogram of the intermediate results in sequences of 3 measurements for which the first and the last yield $n=3$. The single peak reveals that, at the intermediate measurement time, the field contains exactly 3 photons. We use such single photon number peaks to calibrate the phase shifts $\Phi(n)$.

Although the above method allows us to prepare Fock states and to observe qualitatively their evolution, it lacks the resolution required for a precise time analysis. The interval between two measurements $(26 \mathrm{~ms})$ is longer than the lifetime of $n=7\left(T_{c} / 7=18 \mathrm{~ms}\right)$. We can however analyze the data in a more efficient way, making better use of our atomic detection rate. On a single field realiza- tion, each atom detected along direction $\phi$ provides one bit of information $j$ ( $j=0$ for $e$ and $j=1$ for $g$ ). After detecting $N$ atoms, our knowledge of the field is described by an inferred photon number probability distribution $p_{N}^{i}(n)$ linked to the initial distribution $P_{0}(n)$ by Bayes law: $p_{N}^{i}(n)=P_{0}(n) \Pi_{N}(n) / Z$ where $Z$ is a normalization and $\Pi_{N}(n)$ is the product of $N$ functions $p(j, \phi \mid n)$, each describing the information provided by one atomic detection: $p(j, \phi \mid n)=1 / 2\left[1+(-1)^{j}(A+B \cos [\Phi(n)+\phi])\right]$ $[10,15]$. For successive atoms we use, as in [10], four different $\phi$ values $(-1.74,-0.87,0$ and $0.54 \mathrm{rad})$ chosen so that $p(j, \phi \mid n)$ is nearly maximal for $n=6,7,0$ or 1 , respectively. The values $A=-0.1$ and $B=0.7$ are deduced from a fit of the data shown in Fig. 1(b). They deviate from the ideal values ( 0 and 1 , respectively) due to imperfections of Ramsey pulses. For $N \simeq 100, p_{N}^{i}(n)$ converges to a Dirac peak corresponding to the photon number given by the atomic spin analysis.

Let us call $P_{N}(n)$ the ensemble average $\left\langle p_{N}^{i}(n)\right\rangle$ over many realizations in which the field is initially described by $P_{0}(n)$. As the detection process is QND, we have $P_{0}(n)=P_{N}(n)$ for any $N$. In other terms, $P_{0}(n)$ is a fixed point of the transform $P_{0}(n) \rightarrow\left\langle P_{0}(n) \Pi_{N}(n) / Z\right\rangle$. This property allows us to determine $P_{0}(n)$ by iteration of this transform starting with any initial nonvanishing distribution, for instance the flat one $P_{\mathrm{fl}}(n)=1 / 8$. This method can be applied for determining $P(n, t)$ at any time $t$ by selecting in each sequence the sample of $N$ detected probe atoms starting at this time.

We first reconstruct in this way the evolution of $P(n, t)$ for a coherent field injected by $S$ at $t=0$. A measurement sequence, involving $\simeq 2750$ atoms detected in $650 \mathrm{~ms}$, is repeated 2000 times. We reconstruct $P(n, t)$ with the above procedure using $N=25$ atoms and 20 iterations. At each time $t$, we start the iteration with $P_{\mathrm{fl}}(n)$. The temporal resolution is $\simeq 25 \tau=6 \mathrm{~ms} \ll T_{c} / 7$.

Figure 2(a) presents $P(n, t)$ versus $t$ for $n=0 \ldots 7$ and Fig. 2(b) the evolution of the average photon number $\langle n\rangle=$ $\sum_{n} n P(n, t)$. According to theory, $\langle n\rangle$ is exponentially damped towards an offset corresponding to the blackbody background. The experimental decay (solid black line) is indistinguishable from an exponential fit (thin red line) with a $132 \mathrm{~ms}$ time constant agreeing with the independently determined value of $T_{c}$. The offset yields $n_{b}=0.06$, close to the theoretical value $(0.05)$ of the blackbody field at the cavity temperature, $0.8 \mathrm{~K}$. The insets in Fig. 2(b) present snapshots of $P(n, t)$ at three different times with the corresponding Poisson fits. These histograms show, as theoretically predicted [7], that the photon number statistics remains Poissonian under the effect of damping (at the limit where blackbody effects are negligible). The dotted lines in Fig. 2(a) present a numerical solution of the theoretical rate equation using the above determined values for $T_{c}, n_{b}$ and $\langle n\rangle$ at $t=0$. It is in excellent agreement with the data (solid lines). 


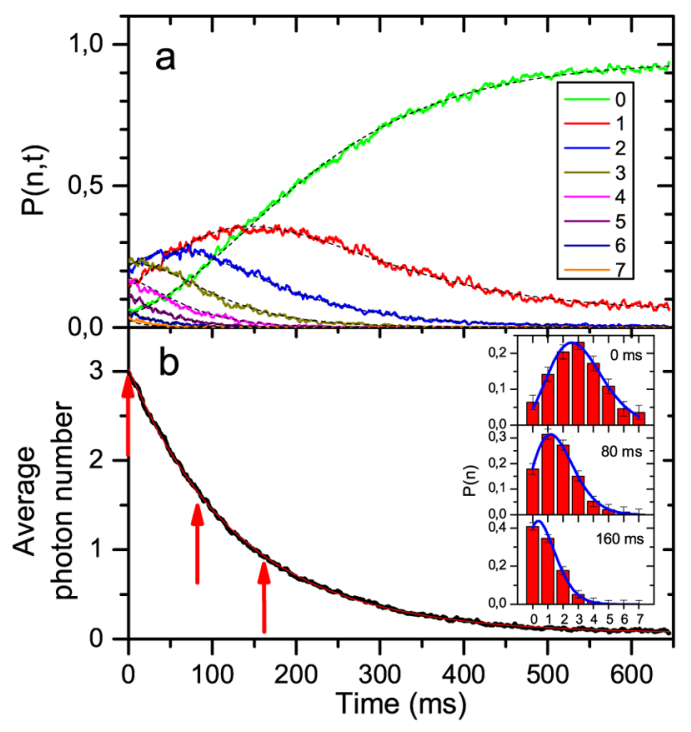

FIG. 2 (color online). Relaxation of a coherent state. (a) Evolution of the photon number probabilities $P(n, t)(n=$ $0 \ldots 7$ according to the color code defined in the inset). Black dotted lines are theoretical. (b) Average photon number $\langle n\rangle$ versus $t$ (solid black line) and exponential fit (thin red line). Insets show the photon number distributions (red histogram) and their Poisson fit (blue lines) at the 3 times shown by arrows.

We go now one step further. By monitoring the decay of selected Fock states, we determine the $K_{n, n^{\prime}}$ coefficients without any a priori assumption about their values. The same experimental data are processed in two steps. First, we analyze separately the 2000 realizations of the experiment in order to select individual Fock states. For each sequence, we compute, after each atom detection, the new inferred photon number distribution $p^{i}(n, t)$ according to Bayes law [10]. We start the analysis of each sequence with the Poisson distribution determined above. Between atoms, we evolve the estimated $p^{i}(n, t)$ according to the theoretical rate equation. This method gives, at each time, the best estimate of the actual photon number distribution in each realization. Except around quantum jumps, $p^{i}(n, t)$ is generally peaked at a single photon number value $n_{0}$. Whenever $p^{i}\left(n_{0}, t\right)>0.7$ we assume that, within a good approximation, the $n_{0}$ Fock state is present in $C$ at this time, which we take as origin $(t=0)$ for subsequent analysis of this Fock state decay.

In a second step, we gather all atomic data following the selection of a given $n_{0}$, obtaining thus ensembles of Fock state-selected field realizations. We apply to each ensemble the iterative analysis described above, reconstructing for each value of $n_{0}$ the subsequent $P_{n_{0}}(n, t)$ distributions. As in the case of a coherent state, we use $N=25$ atoms and 20 iterations starting with a flat initial distribution. Let us stress that this reconstruction procedure does not rely on any theoretical assumption about the form of the relaxation process. We made use of our theoretical knowledge of the $K_{n, n^{\prime}}$ coefficients only in the first step of the data process- ing, in order to optimize the selection of the initial Fock states.

Figure 3 shows in solid lines the reconstructed $P_{n_{0}}(n, t)$ distributions versus time for $n_{0}=0$ to 7 [Figs. 3(a)-3(h)]. In each frame, $P_{n_{0}}\left(n_{0}, t\right)$ is, as expected, maximum at $t=$ 0 , this initial value giving the fidelity of the Fock state selection procedure. The other $P_{n_{0}}(n, 0)$ values are small [16]. At long time $(400 \mathrm{~ms})$ the most probable photon number is always $n=0$, reflecting the irreversible evolution of the field toward the thermal background close to vacuum. For $n_{0}=0$ [Fig. 3(a)] $P_{0}(0, t)$ decreases slightly below 1 , while $P_{0}(1, t)$ reaches an equilibrium value close to 0.06 . This describes the thermalization of the initially empty cavity. For $n_{0}=1$ [Fig. 3(b)] we observe the exponential decay of $P_{1}(1, t)$, together with the increase of $P_{1}(0, t)$, which describes the damping of a single photon into vacuum [9]. For $n_{0}>1$ [Figs. 3(c)-3(h)], $P_{n_{0}}\left(n_{0}, t\right)$ decreases exponentially at a rate increasing with $n_{0}$ (damping of the initial Fock state). The $P_{n_{0}}(n, t)$ functions with $n=n_{0}-1, n_{0}-2, \ldots, 1$ exhibit bell-shaped variations. They peak successively, reflecting the cascade of the photon number from $n_{0}$ down to vacuum.

We extract the damping coefficients by fitting the first $20 \mathrm{~ms}$ of these curves to a solution of Eq. (1) with, as free parameters, $K_{n, n^{\prime}}$ and $P_{n_{0}}(n, 0)$. The procedure is iterative. We get a first approximation of $K_{n, n^{\prime}}$ with $n$ and $n^{\prime} \leq 1$ using the data of Figs. 3(a) and 3(b). We then determine the $K_{n, n^{\prime}}$ with increasing indices by including progressively in

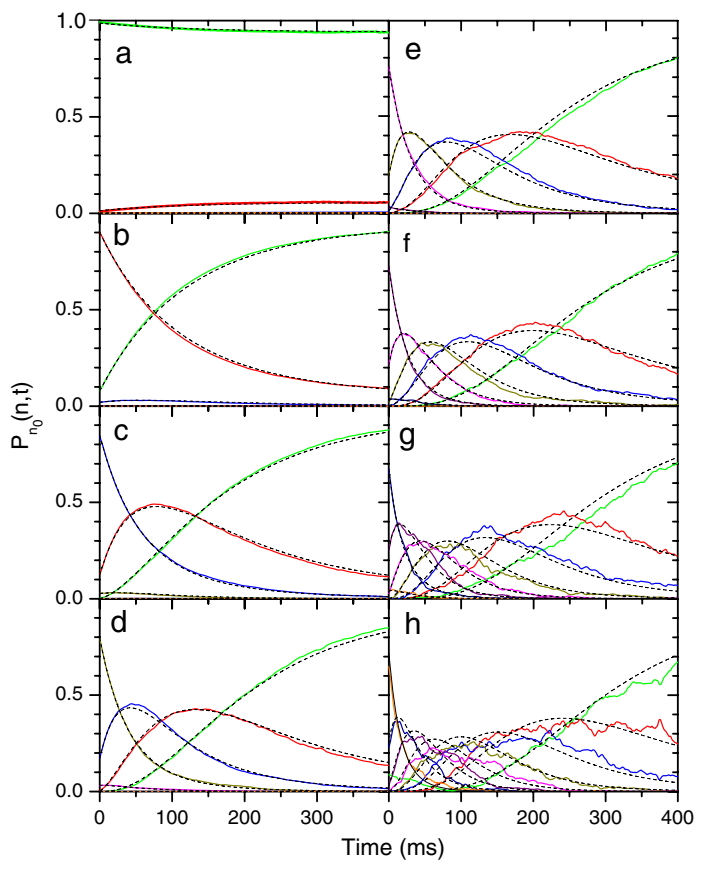

FIG. 3 (color online). Relaxation of Fock states. (a) to (h) Evolution of the photon number distributions $P_{n_{0}}(n, t)$ starting from the Fock states with $n_{0}=0 \ldots 7$ respectively. Same color code as in Fig. 2(a). Dotted black lines are theoretical. 

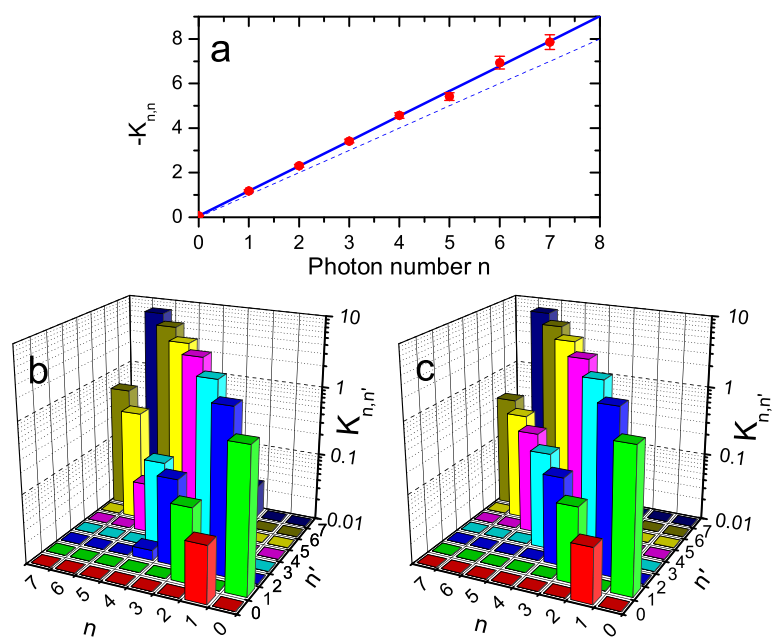

FIG. 4 (color online). Measurement of the photon number probability damping matrix elements $K_{n, n^{\prime}}$. (a) Fock state damping rate $-K_{n, n}$ versus $n$. Circles with error bars are experimental. The solid line gives the theoretical values for $n_{b}=0.06$, the dotted line the expected rates for $n_{b}=0$. (b) $3 \mathrm{D}$ plot of the measured nondiagonal elements $K_{n, n^{\prime}}$ in units of $\kappa$ (log scale). (c) Theoretical 3D plot of $K_{n, n^{\prime}}\left(n \neq n^{\prime}\right)$ in units of $\kappa$ for $n_{b}=$ 0.06 (log scale).

the fits the data of Figs. 3(c)-3(h), optimizing at each step the previously determined parameters.

The obtained $-K_{n, n}$ values, which represent the decay rates of the $n$-Fock states, are shown versus $n$ in Fig. 4(a) (in units of $\kappa$ ). As expected, they vary linearly with $n$. The solid straight line corresponds to the theory for $n_{b}=0.06$, while the dotted line shows the expected variation of $K_{n, n}$ at $T=0 \mathrm{~K}$. This constitutes, together with the work reported in [17], the first measurement of Fock states lifetimes for $n>1$, exhibiting clearly the expected $1 / n$ variation. Moreover, the departure of the experimental points from the dotted line shows that our procedure is precise enough to be sensitive to the small effect of the residual $n_{b}=0.06$ photon blackbody field on the lifetime of Fock states. The nondiagonal $K_{n, n^{\prime}}$ coefficients are shown [Fig. 4(b)] in a 3D plot, in logarithmic scale. The big and small bars near the diagonal correspond to the $K_{n, n+1}$ and $K_{n, n-1}$ coefficients, respectively. The latter, which represent the thermal rates of photon upward jumps, are predicted to vanish for $n_{b}=0$. The logarithmic scale is convenient to display together the $K_{n, n+1}$ and $K_{n, n-1}$ coefficients which differ by about 1 order of magnitude for $n_{b}=0.06$. All other nondiagonal coefficients are 0 within noise. Figure 4(c) shows for comparison the corresponding theoretical coefficients for $n_{b}=0.06$, in excellent agreement with the experiment except for $K_{5,4}$.

The dotted lines in Fig. 3 are the result of a numerical integration of Eq. (1) using the values of $K_{n, n^{\prime}}$ and $P_{n_{0}}\left(n_{0}, 0\right)$ determined by our fit. The excellent agreement with the experiment over the full $400 \mathrm{~ms}$ time interval shows the accuracy of our method.
This Letter demonstrates the power of QND photon number measurements to investigate the quantum behavior of a field stored in a cavity. It probes our understanding of field relaxation and illustrates the sensitivity of large photon number states to decoherence, their lifetime being (at $T=0 \mathrm{~K}$ ) inversely proportional to their energy. The method could be used to monitor the evolution of fields coupled to atomic ensembles used as engineered reservoirs. Combining this method with controlled field displacements leads to a time-resolved quantum state reconstruction [18], which could be used for a determination of the complete decoherence superoperator.

Work supported by Agence Nationale pour la Recherche, Japan Science and Technology Agency, and EU (IP project SCALA). S. D. is funded by Délégation Générale pour l'Armement.

Note added.-While completing this Letter, we learned of a closely related work performed on a superconducting quantum circuit [17].

*brune@lkb.ens.fr

†Present address: ETH Zurich, CH-8093 Zurich, Switzerland.

"Present address: LCFIO, 91127 Palaiseau Cedex, France. ${ }^{\S}$ Present address: Johannes Gutenberg Universität, Institut für Physik, Staudingerweg 7, D-55128 Mainz, Germany.

[1] I. L. Chuang and M. A. Nielsen, J. Mod. Opt. 44, 2455 (1997).

[2] A. M. Childs, I. L. Chuang, and D. W. Leung, Phys. Rev. A 64, 012314 (2001).

[3] M. Howard et al., New J. Phys. 8, 33 (2006).

[4] S. H. Myrskog et al., Phys. Rev. A 72, 013615 (2005).

[5] J. L. O'Brien et al., Phys. Rev. Lett. 93, 080502 (2004).

[6] M. Riebe et al., Phys. Rev. Lett. 97, 220407 (2006).

[7] S. Haroche and J.M. Raimond, Exploring the Quantum (Oxford University Press, Oxford, 2006).

[8] M. Brune et al., Phys. Rev. Lett. 65, 976 (1990).

[9] S. Gleyzes et al., Nature (London) 446, 297 (2007).

[10] C. Guerlin et al., Nature (London) 448, 889 (2007).

[11] N. Lu, Phys. Rev. A 40, 1707 (1989).

[12] M. Hofheinz et al., Nature (London) 454, 310 (2008).

[13] J. M. Raimond, M. Brune, and S. Haroche, Rev. Mod. Phys. 73, 565 (2001).

[14] S. Kuhr et al., Appl. Phys. Lett. 90, 164101 (2007).

[15] Note that in [10] $p(j, \phi \mid n)$ was misprinted. This error had no bearing on the calculations presented there, which were performed with the correct formula.

[16] In Fig. 3(h), $P_{7}(0,0) \simeq 8 \%$ corresponds to 8 photon events, erroneously selected together with $n=7$ and detected as $n=0$ since $\Phi(8) \approx 2 \pi$. This contribution decays rapidly $\left(T_{c} / 8=16 \mathrm{~ms}\right)$. It is negligible for other $n_{0}$ values for which most Fock states have been selected at times much larger than $T_{c} / 8$.

[17] H. Wang et al., preceding Letter, Phys. Rev. Lett. 101, 240401 (2008).

[18] S. Deléglise et al., Nature (London) 455, 510 (2008). 